\title{
Guyanxiao formula antagonizes LPS-induced KOA chondrocyte injury by regulating the SDF-1 / CXCR4 signaling pathway1
}

\section{Qi Zhang ( $\sim 240768149 @ q q . c o m$ )}

Guiyang College of Traditional Chinese Medicine https://orcid.org/0000-0001-7029-4268

\section{Zheng Shu-guang}

Guiyang College of Traditional Chinese Medicine

\section{Wang Bao-juan}

Guiyang College of Traditional Chinese Medicine

Wang Lin

Guiyang College of Traditional Chinese Medicine

\section{Li Tian-yang}

Guiyang College of Traditional Chinese Medicine

\section{Research}

Keywords: Knee osteoarthritis, chondrocytes, Guyanxiao formula, SDF-1 / CXCR4 signaling pathway, Matrix metalloproteinase, Vascular endothelial growth factor

Posted Date: August 7th, 2020

DOl: https://doi.org/10.21203/rs.3.rs-49945/v1

License: (c) (i) This work is licensed under a Creative Commons Attribution 4.0 International License. Read Full License 


\section{Abstract}

Background To determine whether Guyanxiao formula protects chondrocytes in a model of knee arthritis induced by lipopolysaccharide, and whether it can repair chondrocyte damage and suppress osteoarthritis cartilage degeneration by regulating SDF-1 / CXCR4 signaling pathway.

Methods Lipopolysaccharide(LPS) induces chondrocytes in vitro to prepare knee osteoarthritis model. Toluidine blue (TBS) staining was used to observe the changes of proteoglycan content of rabbit chondrocytes in order to identify the source of cells. The biochemical detection method was used to determine the content of inflammatory factor nitric oxide (NO) in chondrocytes to identify whether the osteoarthritis chondrocytes were successfully modeled in vitro.The cell proliferation rate was measured by the cell viability test (CCK-8), the concentration with no obvious cytotoxicity was screened, and the low, medium and high dose groups of Guyanxiao formula were established.Immunofluorescence(IF) staining was used to observe the effect of Guyanxiao formula on the content of type $₫$ collagen in chondrocytes of knee osteoarthritis.Enzyme-linked immunosorbent assay was carried out to determine the expression of inflammatory factors MMP-3, MMP-9 and MMP-13. The mRNA and protein expressions of SDF-1, CXCR4, Vascular endothelial growth factor(VEGF) were analyzed by reverse transcription quantitative polymerase chain reaction and Western blot analysis.

Results The identify of chondrocytes was confirmed with toluidine blue staining. LPS treatment remarkably increased the NO content, indicating successful noodling of the KOA chondrocyte model. According to the CCK-8 experiment results, $0.36,3.6$, and $36 \mu \mathrm{g} / \mathrm{mL}$ were set as the low, medium, and high dose administration concentrations of ostitis.Immunofluorescence(IF) staining showed that the degree of type $\otimes$ collagen damage in each treatment group was improved compared with the model group, and the high concentration group was the most obvious improvement in the Guyanxiao formula treatment group.The levels of MMP-3,MMP-9, MMP-13, and IL-1b were much lower in the Cell supernatant of the each treatment group than in that of model group.The levels of SDF-1, CXCR4, VEGF mRNA and protein were much lower in the Chondrocytes of the each treatment group than in that of model group. In addition, the therapeutic effect of Guyanxiao formula treatment group decreased in a concentrationdependent manner.

Conclusion Guyanxiao formula antagonizes LPS-induced KOA chondrocyte injury by regulating the SDF-1 / CXCR4 signaling pathway.

\section{Background}

Knee osteoarthritis (KOA) is a chronic non-specific osteoarticular disease of high incidence caused by multiple factors. It takes the degenerative disease of the knee articular cartilage as the core, which often involves the synovium, articular cartilage, and other periarticular tissues. In recent years, the disease has been reported to occur frequently in middle-aged and elderly people, with the characteristics of the long course, easy repetition, difficult recovery, and multiple complications ${ }^{(1)}$. Even with the rapid development 
of medical technology, KOA remains to be the fourth general disability disease and a massive problem in the world. To date, no specific drugs or treatment methods have been developed, making it a social public problem affecting human health ${ }^{(2)}$.

The treatment of KOA is mainly divided into three categories: non-drug treatment, drug treatment, and surgical treatment ${ }^{(3-4)}$. However, every treatment has its limitations and shortcomings. Drug treatment, such as long-term use of NSAIDs, causes nausea, vomiting, and other gastrointestinal side effects. Surgical treatment cannot fully treat KOA: postoperative pain, functional exercise, plant life, prosthesis revision, and other issues requires much energy and time ${ }^{(5)}$. Currently, no specific intervention or control measure has been found for the treatment of KOA at the domestic and international levels. The available interventions only alleviate symptoms for the short-term but stop the pathological process of KOA. It is also impossible to reverse the degeneration of articular cartilage.

The pathogenesis of KOA is complex and is poorly understood. It involves multiple signaling pathways. One pathway cannot fully explain the pathogenesis of KOA, and neither can KOA be treated by blocking a single signaling pathway ${ }^{(6)}$. The research on the pathogenesis of KOA and the development of new effective drugs have become a research hotspot. SDF-1 / CXCR4 signaling pathway, one of the most studied signaling pathways for KOA, is closely related to matrix metalloproteinase(MMPs) and vascular endothelial growth factor(VEGF) and plays an essential role in the development of KOA.

Traditional medicine acts as a promising alternative option for the therapy of KOA. Guyanxiao formula is a folk prescription from Qiannan and Qiandongnan region of Guizhou Province, China. It has proved to have good anti-inflammatory and analgesic effects in previous clinical treatment ${ }^{(7-8)}$.Our research team examined the relationship between SDF-1 / CXCR4 signaling pathway and KOA-related inflammatory factors, as well as its expression mechanism on a series of whole animal experiments. They found Guyanxiao formula to effectively inhibit the degradation of the extracellular matrix of knee joint cartilage in vivo. However, the exact mechanism of the Guyanxiao formula and its drug effects are still not well established.

In this study, the rabbit knee joint chondrocytes were cultured in vitro. The KOA model cells were induced by LPS and intervened with the extract of Guyanxiao formula extract. By observing the changes of inflammation indexes, and the expression of the transmission medium related to the SDF-1/CXCR4 pathway, the damaging effect of LPS on chondrocytes was analyzed. Deeper probes into the therapeutic effect and mechanism of Guyanxiao formula on KOA were also discussed.

\section{Materials And Methods}

\section{Cell Culture and Modeling}

Rabbit primary articular chondrocytes (Wuhan Buffal Biotechnology Service Co., Ltd.) were inoculated into a $25 \mathrm{~cm}^{2}$ culture bottle, and DMEM / F-12 containing 10\% fetal bovine serum added. The culture 
flask was placed in a $37^{\circ} \mathrm{C}, 5 \% \mathrm{CO}_{2}$ incubator with regular replacement of the medium. The thirdgeneration cells with good growth conditions were selected for further analysis. The cell concentration was adjusted to $2.5 \times 10^{5}$ cells / $\mathrm{mL}$ when the cells reached the logarithmic growth phase. The model of inflammatory chondrocytes was made by using $8 \mu \mathrm{g} / \mathrm{mL}$ LPS to induce third-generation chondrocytes (up to $80 \%$ ) in the logarithmic growth phase for $24 \mathrm{~h}^{(9)}$.

\section{Toluidine Blue Stain (tbs)}

The morphology, cell growth, and cell density of chondrocytes were observed using an inverted microscope the whole time. After the third-generation rabbit articular chondrocytes attained $90 \%$ growth, they were digested, centrifuged, and then dissociated into cell suspension. The cell concentration was adjusted to $2.5 \times 10^{5}$ cells / $\mathrm{mL}$ and inoculated into a 12-well plate. A coverslip was placed in advance in the well plate, and $1 \mathrm{~mL}$ cell suspension was added into each well. The cells were divided into normal group and LPS model group. The two groups were simultaneously incubated at $37{ }^{\circ} \mathrm{C}$ and $5 \% \mathrm{CO} 2$ for 24 hours. The coverslip was removed from the well plate when the cells had grown to $60-70 \%$ of it. The cells were then fixed with $4 \%$ paraformaldehyde at room temperature for $15 \mathrm{~min}$ and washed three times with PBS. They were then stained with $1 \%$ toluidine blue dyeing solution (pre-heated at $60{ }^{\circ} \mathrm{C}$ ) for $40 \mathrm{~min}$. This was followed by washing the cells three times with distilled water. The cells were then dehydrated, sealed with a transparent film, observed under a light microscope, and photos were taken.

\section{Biochemical Detection Of Nitric Oxide (no) Content}

The third-generation rabbit articular chondrocytes in the logarithmic growth phase and good growth state were collected, digested, and centrifuged to make a cell suspension. The cell concentration of the cell suspension was adjusted to $2.5 \times 10^{5}$ cells / $\mathrm{mL}$. The cells in the suspension were inoculated into 6-well plate in a volume of $2 \mathrm{ml}$ each and divided into normal group and LPS model group. The two groups were cultured at $37{ }^{\circ} \mathrm{C}$ and $5 \% \mathrm{CO} 2$ incubator for 24 hours. The supernatant was sampled for nitric oxide (NO) quantification using the NO detection kit (Batch No: A012-1-2; Nanjing Jiancheng Biotechnology Co, Ltd.) following the manufacturer's instruction. The NO content was calculated according to the standard curve.

\section{Cell Proliferation Assay (cck-8)}

The third-generation rabbit articular chondrocytes in the logarithmic growth phase and in good condition were harvested, and seeded in 96-well plates at a density of 5000 cells/well and cultured in an incubator with $37^{\circ} \mathrm{C}, 5 \% \mathrm{CO}_{2}$ for $24 \mathrm{~h}$. Once cells adhered to the wall, treatments were added to all cell groups. The specific experimental grouping and treatments are shown in Table 1. Culture plates were placed in an incubator with $5 \% \mathrm{CO}_{2}$ and constant temperature for $24 \mathrm{~h}$. Next, $10 \mu \mathrm{L}$ CCK-8 solution was added into each well, and further incubated for $4 \mathrm{~h}$. The culture plate was removed from the incubator to terminate 
the reaction. The absorbance of each well was measured at $450 \mathrm{~nm}$ using a microplate reader (model: $3001-2152$, Thermo Corporation, USA).

Table 1

CCK-8 assay grouping

\begin{tabular}{|c|c|}
\hline Groups & Treatment method \\
\hline Blank group & Complete medium \\
\hline Normal group & Chondrocytes + Complete medium \\
\hline Model group & Chondrocytes + Complete medium $+8 \mu \mathrm{g} / \mathrm{mL}$ LPS \\
\hline $\begin{array}{l}\text { Guyanxiao formula } \\
\text { Group } 1\end{array}$ & $\begin{array}{l}\text { Chondrocytes + Complete medium + } 8 \mu \mathrm{g} / \mathrm{mL} \text { LPS }+36 \mathrm{ng} / \mathrm{mL} \text { Guyanxiao } \\
\text { formula extract }\end{array}$ \\
\hline $\begin{array}{l}\text { Guyanxiao formula } \\
\text { Group } 2\end{array}$ & $\begin{array}{l}\text { Chondrocytes }+ \text { Complete medium }+8 \mu \mathrm{g} / \mathrm{mL} \text { LPS }+0.36 \mu \mathrm{g} / \mathrm{mL} \text { Guyanxiao } \\
\text { formula extract }\end{array}$ \\
\hline $\begin{array}{l}\text { Guyanxiao formula } \\
\text { Group } 3\end{array}$ & $\begin{array}{l}\text { Chondrocytes }+ \text { Complete medium }+8 \mu \mathrm{g} / \mathrm{mL} \text { LPS }+3.6 \mu \mathrm{g} / \mathrm{mL} \text { Guyanxiao } \\
\text { formula extract }\end{array}$ \\
\hline $\begin{array}{l}\text { Guyanxiao formula } \\
\text { Group } 4\end{array}$ & $\begin{array}{l}\text { Chondrocytes + Complete medium }+8 \mu \mathrm{g} / \mathrm{mL} \text { LPS }+36 \mu \mathrm{g} / \mathrm{mL} \text { Guyanxiao } \\
\text { formula extract }\end{array}$ \\
\hline $\begin{array}{l}\text { Guyanxiao formula } \\
\text { Group } 5\end{array}$ & $\begin{array}{l}\text { Chondrocytes + Complete medium }+8 \mu \mathrm{g} / \mathrm{mL} \text { LPS }+360 \mu \mathrm{g} / \mathrm{mL} \text { Guyanxiao } \\
\text { formula extract }\end{array}$ \\
\hline $\begin{array}{l}\text { Guyanxiao formula } \\
\text { Group } 6\end{array}$ & $\begin{array}{l}\text { Chondrocytes + Complete medium }+8 \mu \mathrm{g} / \mathrm{mL} \text { LPS }+3.6 \mathrm{mg} / \mathrm{mL} \text { Guyanxiao } \\
\text { formula extract }\end{array}$ \\
\hline $\begin{array}{l}\text { Guyanxiao formula } \\
\text { Group } 7\end{array}$ & $\begin{array}{l}\text { Chondrocytes + Complete medium }+8 \mu \mathrm{g} / \mathrm{mL} \text { LPS }+36 \mathrm{mg} / \mathrm{mL} \text { Guyanxiao } \\
\text { formula extract }\end{array}$ \\
\hline
\end{tabular}

The cell growth rate was calculated as follows:

Cell proliferation rate $(\%)=[($ experiment-blank $) /($ normal-blank $)] \times 100 \%$

\section{Cell Grouping And Treatment}

We assessed cell toxicity based on cell proliferation assays from which the drug concentrations without obvious cytotoxicity were assigned to low, medium and high dose of Guyanxiao formula. Glucosamine hydrochloride was selected as the positive control drug applied at the concentration of $150 \mu \mathrm{mol} / \mathrm{L}$ (10). The specific experimental grouping and treatments are shown in Table 2. 
Table 2

Experimental grouping

\begin{tabular}{|ll|}
\hline Groups & Treatment method \\
\hline Normal group & Chondrocytes + Complete medium \\
\hline Model group & Chondrocytes + Complete medium $+8 \mu \mathrm{g} / \mathrm{mL} \mathrm{LPS}$ \\
\hline Positive group & $\begin{array}{l}\text { Chondrocytes + Complete medium }+8 \mu \mathrm{g} / \mathrm{mL} \mathrm{LPS}+150 \mathrm{umol} / \mathrm{L} \\
\text { Glucosamine Hydrochloride }\end{array}$ \\
$\begin{array}{l}\text { Low-does of Guyanxiao } \\
\text { formula }\end{array}$ & $\begin{array}{l}\text { Chondrocytes + Complete medium }+8 \mu \mathrm{g} / \mathrm{mL} \mathrm{LPS}+0.36 \mu \mathrm{g} / \mathrm{mL} \\
\text { Guyanxiao formula extract }\end{array}$ \\
$\begin{array}{l}\text { Middle-does of Guyanxiao } \\
\text { formula }\end{array}$ & $\begin{array}{l}\text { Chondrocytes + Complete medium }+8 \mu \mathrm{g} / \mathrm{mL} \mathrm{LPS}+3.6 \mu \mathrm{g} / \mathrm{mL} \\
\text { Guyanxiao formula extract }\end{array}$ \\
\hline $\begin{array}{l}\text { High-does of Guyanxiao } \\
\text { formula }\end{array}$ & $\begin{array}{l}\text { Chondrocytes + Complete medium }+8 \mu \mathrm{g} / \mathrm{mL} \mathrm{LPS}+36 \mu \mathrm{g} / \mathrm{mL} \\
\text { Guyanxiao formula extract }\end{array}$ \\
\hline
\end{tabular}

\section{Immunofluorescence Staining (IF)}

Each group of chondrocytes was grown in the culture medium with a slide for $24 \mathrm{~h}$ to prepare a cell slide. The slides were fixed with $4 \%$ paraformaldehyde for 15 minutes, and $0.5 \%$ Triton X-100 added to break the membrane. After 20 minutes, normal goat serum was added dropwise to block the slide for 30 minutes at room temperature. Collagen II (1:100 dilution) antibody was added to the cell slide and incubated overnight at $4{ }^{\circ} \mathrm{C}$. The following day, the cell slides were washed with phosphate buffer three times, then the fluorescently labeled goat anti-mouse IgG antibody (1: 100 dilution) added and incubated at room temperature for $1 \mathrm{~h}$. the 4,6-diamino-2-phenylindole (DAPI) was added to the cell slide and incubated in the dark for $5 \mathrm{~min}$ at room temperature. The cell slide was washed, sealed with an antifluorescence quenching agent, observed under a fluorescence microscope and image recorded.

\section{Enzyme-linked Immunosorbent Assay (elisa)}

The chondrocytes were treated with different groups of drugs for 24 hours, and the chondrocyte culture medium of each group aspirated. The culture broth was centrifuged at $3000 \mathrm{r} / \mathrm{min}$ for $10 \mathrm{~min}$, and the supernatant collected. The ELISA assays of cell culture fluid samples were performed according to the manufacturer's recommendations of MMP-3 (Batch No.: m1027923-2), MMP-9 (Batch No.: m1027928-2) and MMP-13 (Batch NO.: m1027921-2) ELISA kits, all from Shanghai Enzyme Biological Co., Ltd. A microplate reader was used to detect the Optical Density (OD) value of each well at $450 \mathrm{~nm}$ wavelength, and calculate the content of MMP-3, 9, 13 in the sample. 


\section{Reverse Transcription-polymerase Chain Reaction (real-time Pcr)}

After 24 hours of intervention with different drugs, chondrocytes in each group were extracted with the Trizol method to detect their purity and integrity. The cDNA was synthesized from total RNA using RT-PCR according to the recommended instructions. The PCR amplification was performed by configuring the reaction system to the following reaction conditions: $50^{\circ} \mathrm{C}$ for $2 \mathrm{~min}, 95^{\circ} \mathrm{C}$ for $10 \mathrm{~min}$; and 40 cycles of $30 \mathrm{sec}$ of $95^{\circ} \mathrm{C}$ and $60^{\circ} \mathrm{C}$. The primers used for the PCR reactions have been presented in Table 3 below. The dissolution curve was drawn using GAPDH as the internal reference gene. The relative expression of the target gene was calculated by the $2^{-} \Delta \triangle \mathrm{Ct}$ method.

Tab. 3 Nucleotide sequences of the primers used for a Reverse transcriptase-polymerase chain reaction.

\begin{tabular}{|c|c|c|c|}
\hline Name & Primer & Sequence & Size \\
\hline \multirow[t]{2}{*}{ Rabbit GAPDH } & Forward & 5'- CAGGGCTGCTTTTAACTCTGG -3' & \multirow[t]{2}{*}{$177 \mathrm{bp}$} \\
\hline & Reverse & 5'- TGGAAGATGGTGATGGCCTT -3' & \\
\hline \multirow[t]{2}{*}{ Rabbit SDF-1 } & Forward & 5'- ATCCTCAACACGCCCAAC -3' & \multirow[t]{2}{*}{$104 b p$} \\
\hline & Reverse & 5'- AGGTACTCCTGAATCCAC -3' & \\
\hline \multirow[t]{2}{*}{ Rabbit CXCR4 } & Forward & 5'- СТCTCCTGCTGACTATTCC -3' & \multirow[t]{2}{*}{$339 b p$} \\
\hline & Reverse & 5'- CAAACTCACATCCTTGCTT -3' & \\
\hline \multirow[t]{2}{*}{ Rabbit VEGF } & Forward & 5'- ATGAACTTTCTGCTTTCTTGGG -3' & \multirow[t]{2}{*}{$120 \mathrm{bp}$} \\
\hline & Reverse & 5'- CACTTCGTGGGGTTTATTGTCT -3' & \\
\hline
\end{tabular}

\section{Western Blot (WB)}

Total protein extraction kits were used to extract the total protein of each group of cells and BCA protein concentration detection kit used to determine protein concentration. The extracted protein supernatant was mixed with $5 \times$ SDS loading buffer (volume ratio: 4:1) and boiled at $100{ }^{\circ} \mathrm{C}$ for $10 \mathrm{~min}$. The proteins were separated by SDS-PAGE gel electrophoresis, transferred to PVDF membrane and sealed with $5 \%$ skim milk for $1 \mathrm{~h}$. After washing, the PVDF membrane was soaked in primary antibody $\beta$-actin (diluted 1: 500), SDF-1 (diluted 1: 200), CXCR4 (diluted 1: 1000) and VEGF (diluted 1: 200) and incubated overnight at $4{ }^{\circ} \mathrm{C}$. After washing the membrane the following day, HRP-labeled goat anti-rabbit secondary antibody (diluted 1: 5000 ) was added and incubated at room temperature for $2 \mathrm{~h}$. The membrane was washed, exposed, and analyzed by ECL color development. 


\section{Data analysis}

Statistical Package for Social Science (SPSS) version 24.0 was used for statistical analysis. All the data were expressed as mean \pm standard deviation. One-way analysis of variance (ANOVA) test was used to compare the means among groups. Furthermore, the multiple comparisons were performed with the least significant difference (LSD) test. The statistical significance of the P-value is less than 0.05 was set.

\section{Results}

\section{Toluidine blue staining of Chondrocytes showed reduced expression of proteoglycan in the model group}

Toluidine blue staining showed that the nuclei of the normal group of third-generation chondrocytes were dark blue spheres with different shapes, such as "paving stone" -like distribution, dense in growth, and good in light transmittance. However, the cytoplasm of the model group induced by LPS was light blue and dispersed, with the positive expression of proteoglycan stained by chondrocytes significantly reduced (As shown in Fig. 1.). Therefore, the third-generation chondrocytes were better suited for this experimental study.

Comparison of NO expression in the chondrocyte culture supernatant of normal group and model group

As shown in Table 4, after induction by LPS, the nitric oxide (NO) content of the model group increased significantly compared to the normal group $(P<0.01)$. This indicates that the OA chondrocyte culture model was successfully established.As shown in Table 4. 
Table 4

Average concentration of NO content $( \pm s, n=3)$ in Normal group and model group chondrocytes.

\begin{tabular}{|lll|}
\hline Groups & A & Vitality \\
\hline Normal group & 1.00 & - \\
\hline Normal group & 0.74 & $0.53 \pm 0.17$ \\
\hline Guyanxiao formula Group 1 & 0.77 & $0.71 \pm 0.12$ \\
\hline Guyanxiao formula Group 2 & 0.83 & $0.78 \pm 0.05^{\# \#}$ \\
\hline Guyanxiao formula Group 3 & 0.90 & $0.89 \pm 0.11^{\# \#}$ \\
\hline Guyanxiao formula Group 4 & 0.98 & $0.95 \pm 0.07^{\# \#}$ \\
\hline Guyanxiao formula Group 5 & 0.89 & $0.69 \pm 0.15$ \\
\hline Guyanxiao formula Group 6 & 0.77 & $0.55 \pm 0.07$ \\
\hline Guyanxiao formula Group 7 & 0.69 & $0.37 \pm 0.06$ \\
\hline Note: Compared with the model group, ${ }^{\# \#} \mathrm{P} \leq 0.01$ \\
\hline
\end{tabular}

\section{Screening of low, medium and high concentrations of Guyanxiao formula by CCK-8 experiment}

In the case that the drug action time is 24 hours, the CCK- 8 method was used to detect the cell viability of each experimental group. The value-added ability was significantly reduced in the model group than the normal group $(P<0.01)$. Compared with the model group, the cell proliferation ability of the Guyanxiao formula groups 2,3 , and 4 were restored with group 4 having the best recovery effect $(P \leq 0.01)$. However, the cell viability values of the Guyanxiao formula groups 5,6 , and 7 significantly decrease, showing cytotoxicity to chondrocytes. There was no statistically significant difference in the rate of cell proliferation between the two groups of Guyanxiao formula groups 2,3 , and $4(P>0.05)$, as shown in Table 5. Therefore, $0.36,3.6$, and $36 \mu \mathrm{g} / \mathrm{mL}$ were selected as the experimental concentrations for osteomyelitis reduction. As shown in Table 5. 
Table 5

Effects of different concentrations of Guyanxiao formula on the viability of LPS-induced injured chondrocytes $( \pm \mathrm{s}, n=3)$

\begin{tabular}{|c|c|c|c|}
\hline Groups & MMP-3(pg/mL) & MMP-9(pg/mL) & MMP-13(pg/mL) \\
\hline Normal group & $0.24 \pm 0.02$ & $30.18 \pm 5.89$ & $278.22 \pm 35.93$ \\
\hline Model group & $1.23 \pm 0.05^{\# \#}$ & $172.31 \pm 11.07^{\# \#}$ & $845.93 \pm 76.47^{\# \#}$ \\
\hline Positive group & $0.39 \pm 0.02^{\star \star}$ & $49.58 \pm 4.06^{\star \star}$ & $393.91 \pm 31.65^{\star \star}$ \\
\hline Low-dose of Guyanxiao formula & $1.06 \pm 0.09 * \star$ & $132.64 \pm 11.79 * *$ & $676.81 \pm 41.35^{\star \star}$ \\
\hline Middle-dose of Guyanxiao formula & $0.73 \pm 0.07 * \star$ & $81.29 \pm 5.10 * \star$ & $552.96 \pm 44.63^{\star \star}$ \\
\hline High-dose of Guyanxiao formula & $0.56 \pm 0.03^{* *}$ & $57.10 \pm 4.91 * \star \triangle \triangle$ & $442.91 \pm 20.40 * \star \Delta \triangle$ \\
\hline \multicolumn{4}{|c|}{ Note: Compared with the normal group, ${ }^{\# \# ~} \mathrm{P}<0.01$; compared with the model group, ${ }^{\star *} \mathrm{P}<0.01$; } \\
\hline compared with the positive group, & $P>0.05$ & & \\
\hline
\end{tabular}

\section{Immunofluorescence staining to observe the content of type $₫$ collagen}

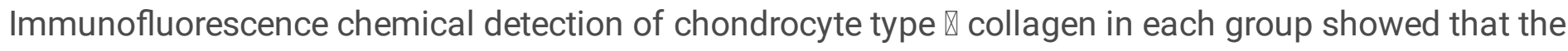
COL- $\$ staining of the model group was a weak positive, which was significantly lower than that of the other 5 groups. The normal group, positive group, and Guyanxiao treatment groups were all positive. A significantly higher expression of COL- $\nabla$ was observed in the positive drug ammonia sugar group and Guyanxiao treatment group than in the model group.As shown in Fig. 2.

\section{Comparison of the contents of MMP-3, MMP-9, and MMP-13 in the chondrocyte culture supernatant}

ELISA detected the expression levels of MMP-3, MMP-9 and MMP-13 in the chondrocyte culture fluid of each group; the results have been shown in Table 6. The expression of MMP-3, MMP-9, MMP-13 increased more in the model group than in the normal group $(P<0.01)$. The expression of MMP-3, MMP-9, MMP-13 in each treatment group significantly decreased compared with the model group $(P<0.01)$. This showed that the Guyanxiao formula had an inhibitory effect on their expressions. There was no significant difference in the expression of MMP-9 and MMP-13 between the positive group and the Highdose of Guyanxiao formula group $(P>0.05)$. The therapeutic effect of the Guyanxiao treatment group depended on its concentration.As shown in Table 6. 
Table 6

Concentration of MMP-3, MMP-9, MMP-13 and NO in chondrocyte supernatant of each group $( \pm$ $\mathrm{s}, n=3$ )

\begin{tabular}{|c|c|c|c|}
\hline Groups & SDF-1 mRNA & CXCR4 mRNA & VEGF mRNA \\
\hline Normal group & $0.96 \pm 0.07$ & $1.06 \pm 0.10$ & $0.97 \pm 0.06$ \\
\hline Model group & $3.18 \pm 0.09^{\# \#}$ & $3.63 \pm 0.16^{\# \#}$ & $4.25 \pm 0.06^{\# \#}$ \\
\hline Positive group & $1.38 \pm 0.07 \star \star$ & $1.32 \pm 0.06^{\star \star}$ & $1.55 \pm 0.11^{\star \star}$ \\
\hline Low-dose of Guyanxiao formula & $2.73 \pm 0.13^{\star \star}$ & $3.01 \pm 0.15^{\star \star}$ & $3.42 \pm 0.06^{\star \star}$ \\
\hline Middle-dose of Guyanxiao formula & $2.13 \pm 0.15^{\star \star}$ & $1.74 \pm 0.08^{\star \star}$ & $2.47 \pm 0.15^{\star \star}$ \\
\hline High-dose of Guyanxiao formula & $1.71 \pm 0.10 * \star$ & $1.55 \pm 0.07 * \star$ & $1.81 \pm 0.04^{\star \star}$ \\
\hline \multicolumn{4}{|c|}{ Note: Compared with the normal group, ${ }^{\# \# ~} \mathrm{P}<0.01$; compared with the model group, ${ }^{* *} \mathrm{P}<0.01$; } \\
\hline \multicolumn{4}{|c|}{ compared with the positive group, $\Delta \Delta P>0.05$} \\
\hline
\end{tabular}

\section{Expression levels of SDF-1, CXCR4 and VEGF mRNA in chondrocytes}

Real-time PCR was used to detect the expression levels of SDF-1, CXCR4, and VEGF mRNA in chondrocytes of each group, the results are shown in Fig. 3 and Table 7. The expression of SDF-1, CXCR4 and VEGF mRNA in the model group increased $(P<0.01)$; The expression levels of SDF-1, CXCR4, and VEGF mRNA in the chondrocytes of each treatment group were suppressed $(P<0.01)$, and the therapeutic effect of Guyanxiao treatment group was dependent on the concentration.As shown in Fig. 3, Table 7.

Table 7

SDF-1, CXCR4, VEGF, IL-1 $\beta$ mRNA expression of chondrocytes in each group $( \pm s, n=3)$

\begin{tabular}{|c|c|c|c|}
\hline Groups & SDF-1/GAPDH & CXCR4/GAPDH & VEGF/GAPDH \\
\hline Normal group & $0.18 \pm 0.01$ & $0.13 \pm 0.02$ & $0.24 \pm 0.04$ \\
\hline Model group & $0.66 \pm 0.09^{\# \#}$ & $0.59 \pm 0.07^{\# \#}$ & $0.83 \pm 0.03^{\# \#}$ \\
\hline Positive group & $0.27 \pm 0.05^{\star \star}$ & $0.24 \pm 0.03^{\star \star}$ & $0.30 \pm 0.02^{\star \star}$ \\
\hline Low-dose of Guyanxiao formula & $0.57 \pm 0.07 \triangle \triangle$ & $0.55 \pm 0.05^{\triangle} \triangle$ & $0.71 \pm 0.03^{\star *}$ \\
\hline Middle-dose of Guyanxiao formula & $0.47 \pm 0.01^{\star \star}$ & $0.42 \pm 0.02^{\star \star}$ & $0.60 \pm 0.02 * \star$ \\
\hline High-dose of Guyanxiao formula & $0.39 \pm 0.03^{\star \star}$ & $0.32 \pm 0.02^{\star \star}$ & $0.47 \pm 0.05^{\star \star}$ \\
\hline \multicolumn{4}{|c|}{ Note: Compared with the normal group, ${ }^{\# \#} \mathrm{P}<0.01$; compared with the model group, $* \star P<0.01$} \\
\hline
\end{tabular}


Western Blot detected the expression of SDF-1 protein, CXCR4 protein, and VEGF protein in the cartilage group of each group, and the results are shown in Fig. 3 and Table 8. The expression levels of SDF-1, CXCR4, and VEGF in the chondrocytes in the model group were up-regulated and suppressed in the treatment group $(P<0.01)$. The therapeutic effect of the Guyanxiao treatment group decreased by a decrease in concentration.As shown in Fig. 4 and Table 8.

Table 8

Content of SDF-1, CXCR4 and VEGF in chondrocytes of each study group $( \pm s, n=3)$

\begin{tabular}{|c|c|c|c|}
\hline Groups & SDF-1/GAPDH & CXCR4/GAPDH & VEGF/GAPDH \\
\hline Normal group & $0.18 \pm 0.01$ & $0.13 \pm 0.02$ & $0.24 \pm 0.04$ \\
\hline Model group & $0.66 \pm 0.09^{\# \#}$ & $0.59 \pm 0.07^{\# \#}$ & $0.83 \pm 0.03^{\# \#}$ \\
\hline Positive group & $0.27 \pm 0.05^{\star \star}$ & $0.24 \pm 0.03^{\star *}$ & $0.30 \pm 0.02^{\star \star}$ \\
\hline Low-dose of Guyanxiao formula & $0.57 \pm 0.07 \triangle \triangle$ & $0.55 \pm 0.05 \triangle \triangle$ & $0.71 \pm 0.03^{\star \star}$ \\
\hline Middle-dose of Guyanxiao formula & $0.47 \pm 0.01 * \star$ & $0.42 \pm 0.02^{\star \star}$ & $0.60 \pm 0.02 * \star$ \\
\hline High-dose of Guyanxiao formula & $0.39 \pm 0.03^{\star \star}$ & $0.32 \pm 0.02 * \star$ & $0.47 \pm 0.05^{\star \star}$ \\
\hline \multicolumn{4}{|c|}{ Note: Compared with the normal group, ${ }^{\# \#} \mathrm{P}<0.01$; compared with the model group, ${ }^{* *} \mathrm{P}<0.01$} \\
\hline
\end{tabular}

\section{Discussion}

Knee osteoarthritis (KOA) is a chronic disease whose main pathological change is the destruction of the cartilage function of the knee joint. Articular cartilage is mainly composed of chondrocytes and extracellular matrix. Chondrocytes account for about $2 \%$ of cartilage volume, and its main role is to maintain normal cartilage metabolism and secrete extracellular matrix ${ }^{(11)}$. They are highly differentiated cells whose main secretions are collagen (mainly type II collagen) and proteoglycans. The synthesis and degradation of the extracellular matrix (ECM) have been in a dynamic equilibrium state. When a variety of pathogenic factors is causing the inflammation, the balance mechanism is destroyed, leading to chondrocyte apoptosis, extracellular matrix degradation, degeneration of cartilage tissue, and eventually joint cartilage degradation results to the occurrence and development of osteoarthritis ${ }^{(12)}$. Proteoglycan, a matrix component secreted from chondrocytes, is the main identification index of chondrocyte structure and function. It is a type of carbohydrate complex composed of a core protein and one or more covalently linked amino polysaccharides. The amino polysaccharide was stained with toluidine blue staining solution to identify the experimental cells confirming that the experimental operating cells were articular chondrocytes.

Lipopolysaccharide (LPS) as a complex is composed of polysaccharide and lipoid A by a covalent bond. It is also a common proinflammatory factor, which has been applied in various in vitro studies of 
inflammation ${ }^{(13)}$. This study confirmed that the treatment of chondrocytes with LPS decreased viability and slowed down their rate of proliferation. This was consistent with the pathological changes of chondrocytes during the pathogenesis of osteoarthritis, where proliferative activity decreases and apoptosis increases. In this study, LPS-induced OA chondrocytes were used as the object of administration to explore the mechanism of inflammatory factors in the pathogenesis of KOA. The pathological mechanism of osteoarthritis is mainly related to the inflammatory reaction in cartilage tissue. Nitric Oxide (NO) is an important inducer of chondrocyte apoptosis and plays an important role in the pathogenesis of KOA. During the KOA attack, NO synthase can promote chondrocytes to produce large amounts of NO, thereby inducing apoptosis to participate in the pathological development of KOA. This study found an increase in the expression of NO in chondrocytes treated in vitro by LPS, which was consistent with the characteristics of inflammatory response. Therefore, the in vitro inflammatory chondrocyte model established in this experiment simulated the pathogenesis of osteoarthritis.

Stromal cell-Derived Factor1(SDF-1) is a type of cytokine that causes leukocyte metastasis or inflammatory response. It is the only known chemokine that can bind to and activate $\mathrm{C}-\mathrm{X}-\mathrm{C}$ motif chemokine receptor4 (CXCR4) on the surface of chondrocyte. The combination of the two can form a coupled molecular pair that is closely related to the transmission of information between cells and cell migration ${ }^{(14)}$. Exogenous chemokines from the synovium and bone marrow belong to the $C-X-C$ chemokine subfamily, which is a very strong inducer of cartilage matrix degradation. For patients with osteoarthritis, SDF-1 is a super powerful chemoattractant factor present in lymphocytes and monocytes, which can induce its transmembrane chemotaxis. The SDF-1 plays an important role in the development of articular cartilage degeneration in patients with osteoarthritis ${ }^{(15-16)}$. The SDF-1/CXCR4 signaling pathway is closely related to inflammation, cell growth, and oxidative damage. It can induce the release of downstream inflammatory factors and play an important role in the process of inflammatory cell infiltration, cell migration, and organ development ${ }^{(17)}$. Recent studies have found that when OA occurs, this signal pathway is over-activated, resulting in a significantly higher SDF-1 content in the synovial fluid, which is significantly reduced after blockage by antagonists ${ }^{(18-19)}$. The results showed that the expression levels of SDF-1, CXCR4 genes, and proteins in KOA chondrocytes treated with Guyanxiao formula were significantly reduced suggesting that Guyanxiao formula could play an anti-inflammatory effect by inhibiting the activation of the SDF-1/CXCR4 signaling pathway and improving the microenvironment in cartilage tissue to achieve the purpose of treating KOA.

Vascular endothelial growth factor (VEGF) is the most important cytokine that induces angiogenesis ${ }^{[20]}$. Numerous studies ${ }^{(21-24)}$ have reported over-expression of VEGF in the synovial tissue and cartilage tissue of KOA patients. Besides, according to the Mankin histological score, the OA histological grade is positively correlated with the expression level of VEGF. It has been found that in the late stage of OA, the expression of VEGF in human chondrocytes has an upward trend. This is because VEGF can induce the formation of new blood vessels at the junction, destroy the local cartilage, and stimulate the growth of inflammatory cells due to plasma extravasation, further aggravating the inflammation of osteoarthritis. It can be inferred that VEGF plays an important role in the formation of synovial neovascularization, the 
chemotaxis of inflammatory cells to the inflammation site, and the moisturizing. VEGF is one of the important mechanisms involved in the development of OA. The VEGF is located downstream of the SDF$1 / C X C R 4$ signaling pathway and can be activated by the pathway. It is an important transcription factor between SDF-1/CXCR4 and matrix metalloproteinase (MMP). Krycze ${ }^{(25)}$ found that VEGF and CXCR4 can interact to promote the indirect migration of endothelial cells mediated by SDF-1. By suspending the SDF$1 / C X C R 4$ signaling pathway, VEGF can reduce the angiogenesis attached to it, suggesting that the signaling pathway is directly or indirectly involved in angiogenesis when it exerts its biological function. With the addition of VEGF inhibitors, the expression levels of SDF-1 and CXCR4 showed a significant reduction, indicating that VEGF can regulate the SDF-1/CXCR4 signaling pathway ${ }^{(26)}$. The results found that the expressions of SDF-1, CXCR4, and VEGF in the model group were significantly increased, revealing that the three signaling factors play a vital role in accelerating the process of KOA. Following the intervention of the Guyanxiao formula, an obvious reduction of these signaling factors was seen, indicating that Guyanxiao formula may inhibit the expression of VEGF and block the binding of SDF-1 to its receptor CXCR4, thereby delaying the development of OA and achieving the purpose in treating KOA.

Matrix metalloproteinases (MMPs) are zinc ion-dependent protease superfamilies that can degrade substrates other than polysaccharides. The MMP, secreted by chondrocytes, is an important inflammatory factor mediating cartilage destruction of osteoarthritis and proteolytic enzyme involved in the destruction of the extracellular matrix. Studies have confirmed that the combination of SDF-1 and CXCR4 induces bone cells to secrete MMPs, and the expression of MMPs increases during the degradation of KOA cartilage, resulting in further destruction of articular cartilage ${ }^{(27)}$. MMP-3 is produced by connective tissue cells, synovial cells, and fibroblasts. It has the strongest ability to degrade proteoglycans and induce the inflammatory production of other MMPs. It can also degrade collagen, reduce the stability of the matrix, and the external resistance of cartilage resulting in the damage of articular cartilage. The concentration of MMP-3 can directly reflect the degree of joint damage ${ }^{(28)}$. MMP-9 is a gelatinase, which is secreted by chondrocytes, neutrophils, osteoclasts, and the other cells. It is mainly expressed in the deeper layer of OA cartilage, and has an important impact on remodeling subchondral bone and promoting angiogenesis. It can also reflect the degree of joint damage ${ }^{(29)}$. MMP-13 is a key enzyme that degrades type II collagen and can activate other MMPs. It plays a key role in the destruction of osteoarthritis cartilage ${ }^{(30)}$.

The zymogen of MMPs has more capabilities after being activated. In addition to degrading the extracellular matrix, it can also activate other MMPs continuously, ensuring the continuation of the vicious cycle to form a chain reaction and cause serious and continuous damage to the extracellular matrix. It also destroys the collagen network and growth environment of cartilage tissue, further aggravating cartilage tissue damage and accelerating the KOA process ${ }^{(31)}$. The SDF-1/CXCR4 signaling pathway and VEGF can not only synergistically promote angiogenesis of OA, but also adjust hematopoietic cells and accelerate the regeneration of blood vessels in ischemic organs. The large increase of neovascularization can also stimulate the release of MMPs to make ECM. Massive degradation breaks the steady-state between ECM synthesis and degradation, altering the metabolic 
microenvironment within the joint. This leads to the destruction of the structural and functional integrity of the articular cartilage; hence, the occurrence and development of $\mathrm{KOA}^{(32)}$. The results showed that the chondrocytes in the model group were induced by lipopolysaccharide, which aggravated the ECM damage, reducing greatly the positive coloration of type II collagen and proteoglycan. The expression levels of MMP-3, $-9,-13$ were significantly increased, suggesting that the expression level of MMPs can directly affect the development of osteoarthritis. The interventional treatment of the Guyanxiao formula significantly reduced the expression of MMP-3, MMP-9, and MMP-13, showing its function of antiinflammatory and negative regulation of SDF-1/CXCR4 signaling pathway.

Under normal circumstances, chondrocytes can secrete a large number of proteoglycans, which can be degraded by MMPs., thus maintaining the circulation and balance of cell support strength. The damaged chondrocytes can secrete excessive amounts of MMPs, resulting in damage to the above equilibrium and inducing osteoarthritis ${ }^{(33)}$. MMPs are important sources of ECM degradation. The main component of biodegradable ECM is type II collagen and proteoglycan. Type II collagen is the major component of cartilage collagen, which maintains the elasticity of cartilage with proteoglycan. In this experiment,

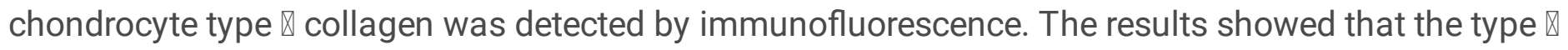
collagen content in the model group was significantly less than in the Guyanxiao formula intervention treatment group. This shows that Guyanxiao formula prescription may promote type $₫$ collagen synthesis, repair the ECM of cartilage, and inhibit the progress of KOA.

Guyanxiao formula is traditional Chinese medicine, which contains a variety of active ingredients. It can be used in multiple ways, acting on multiple targets and affecting multiple links to participate in the treatment of diseases. The active ingredient mutually plays the role of reducing swelling and pain, removing dampness, relaxing muscles and collaterals, and reducing ventilating and bleeding. Preliminary

experiments and clinical studies $(7,8)$ have shown that Guyanxiao formula has obvious therapeutic effects on the treatment of osteoarthritis such as femoral head necrosis and KOA. Its prescription has anti-inflammatory and analgesic effects since it can reduce the release of inflammatory factors of articular cartilage and improve bone tissue structure. After Guyanxiao treatment in this experimental study, the proliferative activity of LPS-induced arthritis chondrocytes increased, and the levels of inflammatory factors MMP-3, MMP-9, MMP-13 in the cell culture supernatant decreased. This shows that the Guyanxiao formula inhibits the release of inflammatory factors from arthritis chondrocytes.

\section{Conclusion}

This study has proved that Guyanxiao formula enhances cell viability, inhibits LPS-induced chondrocyte damage and inflammatory response, and improves the apoptosis of cells. It also enhances the compensatory increase of inflammatory factors such as matrix metalloproteinase by LPS treatment and restores the integrity of the extracellular matrix by maintaining the balance between its synthesis and degradation. Guyanxiao formula achieves its action by reducing the expression of SDF-1 and blocking the SDF-1 / CXCR4 signaling pathway, thereby reducing cell inflammation, inhibiting angiogenesis, and reducing the damage of chondrocytes. The therapeutic effect of the High-dose of Guyanxiao formula on 
some inflammatory indicators is similar to the positive group of glucosamine hydrochloride. This indicates that the Guyanxiao formula has the same therapeutic effect on KOA as modern medicine. This study has found the Guyanxiao formula to be a new target for the treatment of KOA. The study has provided a new direction for the treatment of KOA and outlined an experimental basis for the future research of the mechanism of Guyanxiao formula in improving the symptoms of KOA.

\section{Acronym Meaning}

Table 9

Acronym meaning

\begin{tabular}{|ll|}
\hline Abbreviations & Meaning \\
\hline KOA & knee osteoarthritis \\
\hline MMPs & matrix metall oproteinases \\
\hline ECM & extracellular matrix \\
\hline SDF-1 & stromal cell derivedfactor-1 \\
\hline CXCR4 & chemokine receptor \\
\hline VEGF & vascular endothelial gowth factor \\
\hline LPS & Lipopolysaccharide \\
\hline GLU & Glucosamine hydrochloride \\
\hline
\end{tabular}

\section{Declarations}

\section{Ethical Approval and Consent to participate}

This study was approved by the ethical committee of Laboratory Animal Research Institute of Guizhou University of traditional Chinese Medicine『and this paper does not contain any research on human participants.

\section{Consent for publication}

The author guarantees that all copyright of the text (including reprint, translation, image production, microfilm, electronic production and all similar reproduction) is agreed to contribute to the journal. 
The author solemnly promises that the paper is the original one, and that all or part of the contents of the paper have never been published in any other journal in any form. There is no problem of duplicate contributions, no plagiarism or plagiarism, and no content that violates laws and regulations or infringes upon the rights and interests of others.All data are fully available without restriction.

\section{Competing interests}

The authors declared that they have no conflicts of interest to this work. We declare that we do not have any commercial or associative interest that represents a conflict of interest in connection with the work submitted.

\section{Funding}

This paper is supported by the National Natural Science Foundation of China (NSFC)(Project No.: 81660823, 81960914).

\section{Authors' contributions}

Zheng Shu-guang contributed the central idea; Wang Lin analysed most of the data; Zhang Qi and Wang Bao-juan contributed to refining the ideas and wrote the initial draft of the paper; Li Tian-yang carrying out additional analyses; all authors discussed the results and revised the manuscript.

\section{Acknowledgements}

I am greatly indebted to my supervisor, Professor Zheng Shu-guang, for his valuable instructions and suggestions on my thesis as well as his careful reading of the manuscript.I feel grateful to all the teachers in the College of Guizhou University of traditional Chinese Medicine who once offered me valuable courses and advice during my study. Last but not least, I owe much to my friends and classmates for their valuable suggestions and critiques which are of help and importance in making the thesis a reality.

\section{Authors' information}

Brief introduction of the First author: Zhang Qi (1995 -), female, master's degree, main research direction: basic theory of traditional Chinese medicine, national medicine and osteoarthropathy. E-mail囚 
Corresponding author: Zheng Shu-guang (1966 -), male, Professor, master's supervisor, main research direction: basic theory of traditional Chinese medicine, national medicine and osteoarthropathy, e-mail: 332126632@qq.com.

Author: Wang Bao-juan (1992-), female, master's degree, main research direction: basic theory of traditional Chinese medicine, national medicine and osteoarthropathy. E-mail囚769774370@qq.com; Tel『 15208516782.

Author:Wang Lin (1992-), female, master's degree, main research direction: basic theory of traditional Chinese medicine, national medicine and osteoarthropathy. E-mail『864901324@qq.com; Tel》 18243185166.

Author: Li Tian-yang(1994-),male, master's degree, main research direction: basic theory of traditional Chinese medicine, national medicine and osteoarthropathy. E-mail囚1013287867@qq.com; Tel『 15761638421.

\section{References}

1. Knoop J, van der Leeden M, van der Esch M, de Rooij M, Peter WF, Bennell KL, Steultjens MPM, Hakkinen A, Roorda LD, Lems WF, Dekker J. Is a model of stratified exercise therapy by physical therapists in primary care feasible in patients with knee osteoarthritis?: a mixed methods study[J]. Physiotherapy,2020,03(106):101-110.

2. Tang, Xu,Wang Shengfeng,Zhan Siyan,et al. The Prevalence of Symptomatic Knee Osteoarthritis in China: Results From the China Health and Retirement Longitudinal Study.[J]. Arthritis \& rheumatology,2016,68(3):648-653.

3. Xu Ying F, Kaijian. Wang Tingyu. The pathogenesis of osteoarthritis and the progress of drug treatment [J]. Practical Drugs Clinical Medicine. 2018;21(12):1424-9.

4. Bohensky MA, Ackerman I, Desteiger R, et al. Lifetime Risk of Total Knee Replacement and Temporal Trends in Incidence by Health Care Setting, Socioeconomic Status, and Geographic Location[J]. Arthritis Care \& Research, 2014, 66(3):424-431.].

5. Beswick AD, Wylde V, Gooberman-Hill R,et al. What proportion of patients report long-term pain after total hip or knee replacement for osteoarthritis? A systematic review of Prospective studies in unselected patients[J]. Bmj Open. 2012;2(1):e000435.

6. Zhang JXu,YuanfengChen,YangLiu,Jinfang,Qinglin Kang,Kiwai Ho,Yimin Chai,Gang Li. Effect of SDF1/Cxcr4 Signaling Antagonist AMD3100 on Bone Mineralization in Distraction Osteogenesis[J]. Calcified Tissue International,2017,100 (6):641-652.

7. Wang Xinggui Z, Shuguang Z, Hanhua, et al. Effect of Guizhou Miao fumigation therapy on the expression of IL-1 and TNF-a protein in early KOA rabbit cartilage [J]. Journal of Guiyang College of 
Traditional Chinese Medicine. 2017;39(06):38-42 + 47.

8. Zheng Shuguang Xu, Hang. Xue Linshan.Miao medicine fumigation therapy for early necrosis of femoral head [J]. Chinese Journal of Experimental Prescriptions. 2012;18(09):270-2.

9. Arend WP, Joslin FG, Massoni RJ. Characteristics of chondrocyte responses to a human interleukin 1like factor.[J]. Clinical immunology and immunopathology,1985,36(3):358-370.

10. Cécile B-C,Iveta Garaiova,Sue F, Plummer,John L. Harwood,Bruce Caterson,Clare E Hughes. Glucosamine Hydrochloride but Not Chondroitin Sulfate Prevents Cartilage Degradation and Inflammation Induced by Interleukin-1a in Bovine Cartilage Explants[J]. Cartilage,2016,7(1):70-81.

11. Sun Y, Yan L, Chen S, et al. Functionality of decellularized matrix in cartilage regeneration: $A$ comparison of tissue versus cell sources[J]. Acta Biomater. 2018;74:56-73.

12. Zamli Z, Adams M, Tarlton J, et al. Increased Chondrocyte Apoptosis Is Associated with Progression of Osteoarthritis in Spontaneous Guinea Pig Models of the Disease[J]. Int J Mol Sci. 2013;14(9):17729-43.

13. Zeyu Huang,Virginia Byers Kraus. Does lipopolysaccharide-mediated inflammation have a role in OA?[J]. Nat Rev Rheumatol. 2016;12(2):123-9.

14. RATNESWARAN A, SUN MM. DUPUIS H, et al. Nuclear receptors regulate lipid metabolism and oxidative stress markers in chondrocytes[J]. J Mol Med (Berl). 2017;95(4):431.

15. Kanbe K, Takagishi K, Chen Q. Stimulation of matrix metalloprotease 3 release from human chondrocytes by the interaction of stromal cell-derived factor 1 and CXC chemokine receptor 4[J]. Arthritis rheumatism. 2002;46(1):130-7.

16. Grassi F, Cristino S, Toneguzzi S, et al. CXCL12 chemokine up-regulates bone resorption and MMP-9 release by human osteoclasts: CXCL12 levels are increased in synovial and bone tissue of rheumatoid arthritis patients[J]. J Cell Physiol. 2010;199(2):244-51.

17. Jin D. Cytokine-mediated deployment of SDF-1 induces revascularization through recruitment of CXCR4 + hemangiocytes[J]. Nat Med, 2006, 12.]-[[] Marquez-Curtis L A, Janowska-Wieczorek A. Enhancing the Migration Ability of Mesenchymal Stromal Cells by Targeting the SDF-1/CXCR4 Axis[J]. BioMed Research International, 2013, 2013:1-15.

18. The critical role. of SDF-1/CXCR4 axis in cancer and cancer stem cells metastasis[J]. J Endocrinol Invest. 2008;31(9):809-19.

19. Wu Zhouling B, Shier B, Guifei C. Wenxia. Biological characteristics of stromal cell-derived factor 1 and its $\mathrm{G}$ protein coupled receptor (CXCR4) signal axis [J]. Chinese Journal of Tissue Engineering Research. 2019;23(09):1434-40.

20. Meng Xuhan. Three antagonists targeting in vivo blocking SDF-1 / CXCR4 signaling pathway to regulate articular cartilage degeneration [D]. Kunming Medical University, 2018.

21. Shi Yang L, Bin C Le. Levels and significance of serum vascular endothelial growth factor and matrix metalloproteinase-9 in patients with hepatocellular carcinoma [J]. Journal of Practical Diagnosis and Therapy, 2008, 22 (4): 255-257. 
22. Saetan N, Honsawek S, Tanavalee A, et al. Relationship of plasma and synovial fluid vascular endothelial growth factor with radiographic severity in primary knee osteoarthritis[J]. International Orthopaedics, 2013, 38(5).

23. Tibesku CO, Daniilidis K, Skwara A, et al. Expression of Vascular Endothelial Growth Factor on Chondrocytes Increases with Osteoarthritis - An Animal Experimental Investigation[J]. The Open Orthopaedics Journal. 2011;5(1):177-80.

24. Pufe T, Petersen W, Tillmann B, et al. The splice variants VEGF121 and VEGF189 of the angiogenic peptide vascular endothelial growth factor are expressed in osteoarthritic cartilage[J]. Arthritis Rheum. 2001;44(5):1082-8.

25. Enomoto H, Inoki I, Komiya K, et al. Vascular Endothelial Growth Factor Isoforms and Their Receptors Are Expressed in Human Osteoarthritic Cartilage[J]. Am J Pathol. 2003;162(1):0-181.

26. Kryczek I, Wei S, Keller E, et al. Stroma-derived factor (SDF-1/CXCL12) and human tumor pathogenesis[J]. AJP Cell Physiology. 2007;292(3):C987-95.

27. Zhang Jin Z, Xinyuan Z. Jianhua. Clonidine promotes the proliferation and migration of trophoblast cells based on the regulation of SDF-1 / CXCR4 signal by VEGF [J]. China Medical Herald. 2016;13(15):35-9.

28. Ingale PA, Hadden WA. A Review of Mobile Bearing Unicompartmental Knee in Patients Aged 80 Years or Older and Comparison With Younger Groups[J]. J Arthroplasty. 2013;28(2):262-7.e2.

29. Expression and significance of MMP-3. and MMP-13 in synovium of patients with osteoarthritis [J]. Chinese Journal of Osteoporosis, 2014 (6): 593-596.

30. Yunyun L,Sinkeviciute Dovile,He Yi,Karsdal Morten,Henrotin Yves,Mobasheri Ali,Önnerfjord Patrik,Bay-Jensen Anne. The minor collagens in articular cartilage.[J]. Protein \& cell,2017,8(8):560572.

31. Li Yuejun Z, Weiguo F, Qinzheng Gu, Peilun G, Peng S, Yongzhi Y, Pengfei, Dong Jinbo. Clinical significance of $\beta$-catenin and matrix metalloproteinase 13 in synovial tissue of osteoarthritis [J]. Chinese Journal of Tissue Engineering Research. 2018;22(16):2472-7.

32. Wang CC, Guo L, Tian FD, et al. Naringenin regulates production of matrix metalloproteinases in the knee-joint and primary cultured articular chondrocytes and alleviates pain in rat osteoarthritis model[J]. Brazilian Journal of Medical and Biological Research, 2017, 50(4).

33. Huang Z, Wang L, Guo S, et al. The role of SDF-1/CXCR4 in the vasculogenesis and remodeling of cerebral arteriovenous malformation[J]. Therapeutics and Clinical Risk Management, 2015.

\section{Figures}




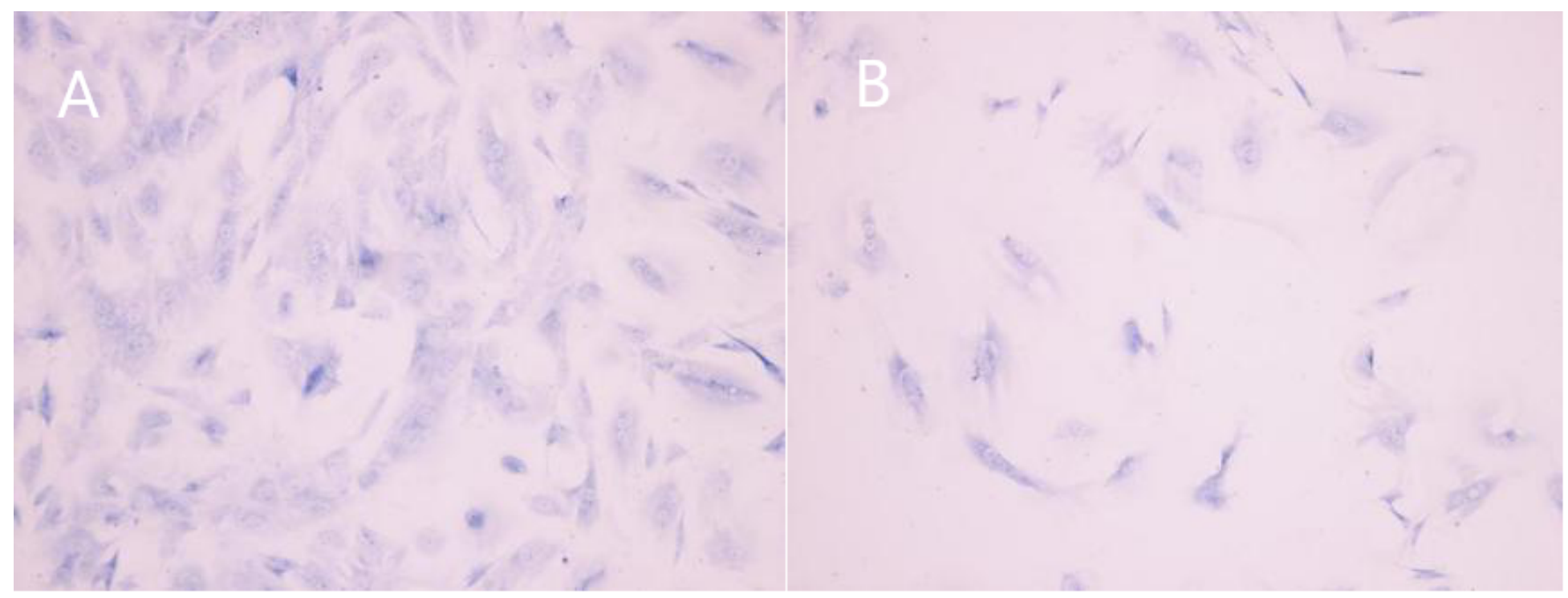

\section{Figure 1}

Toluidine blue staining of normal group and model group under a magnification of $\times 200$ Note: A.Normal group B.Model group
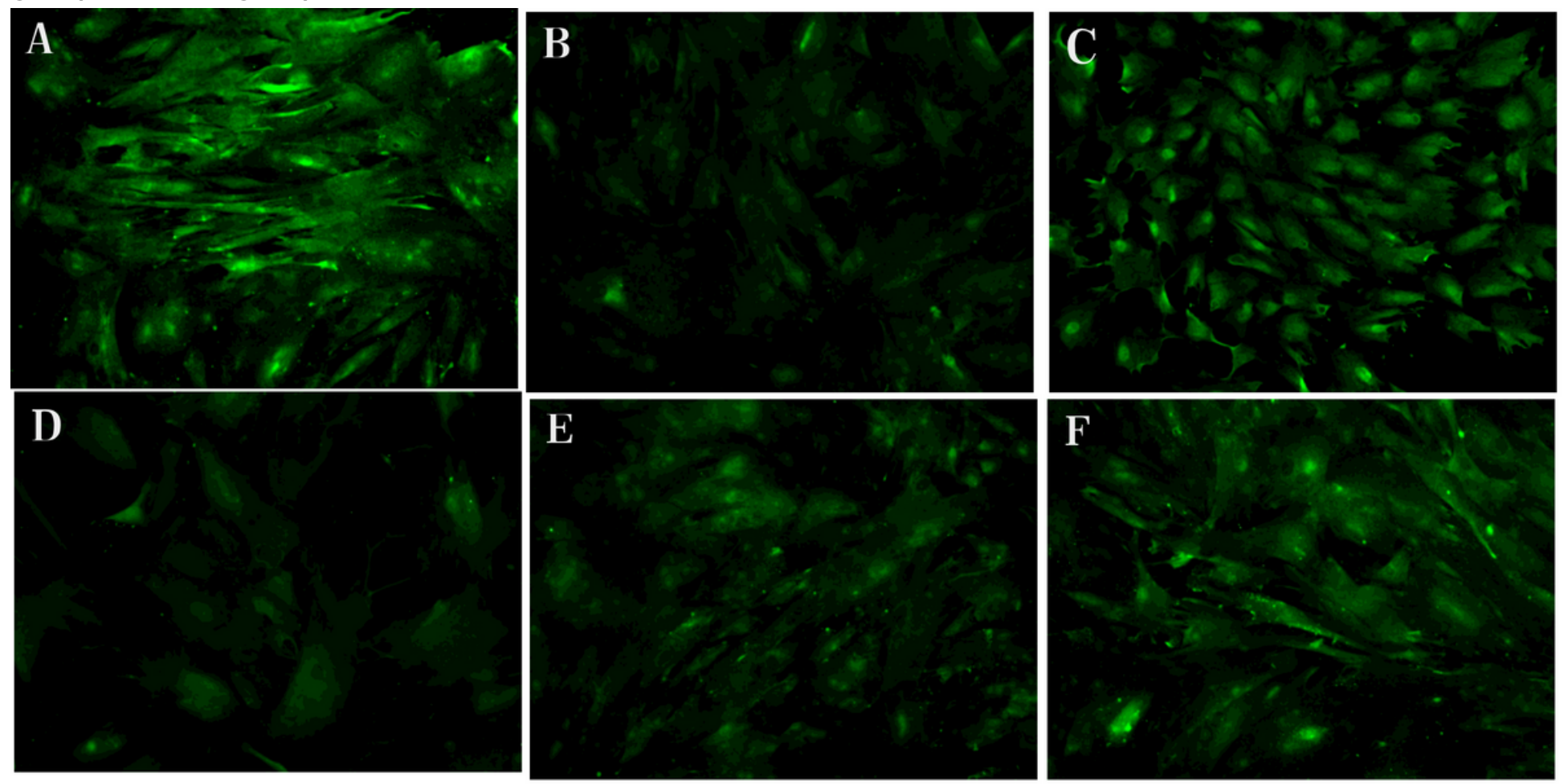

\section{Figure 2}

Results of immunofluorescence staining of chondrocytes in six groups under a magnification of $\times 200$ Note: A.Normal group B.Model group C.Positive group D.Low-dose of Guyanxiao E.Middle-dose of Guyanxiao F.High-dose of Guyanxiao formula 

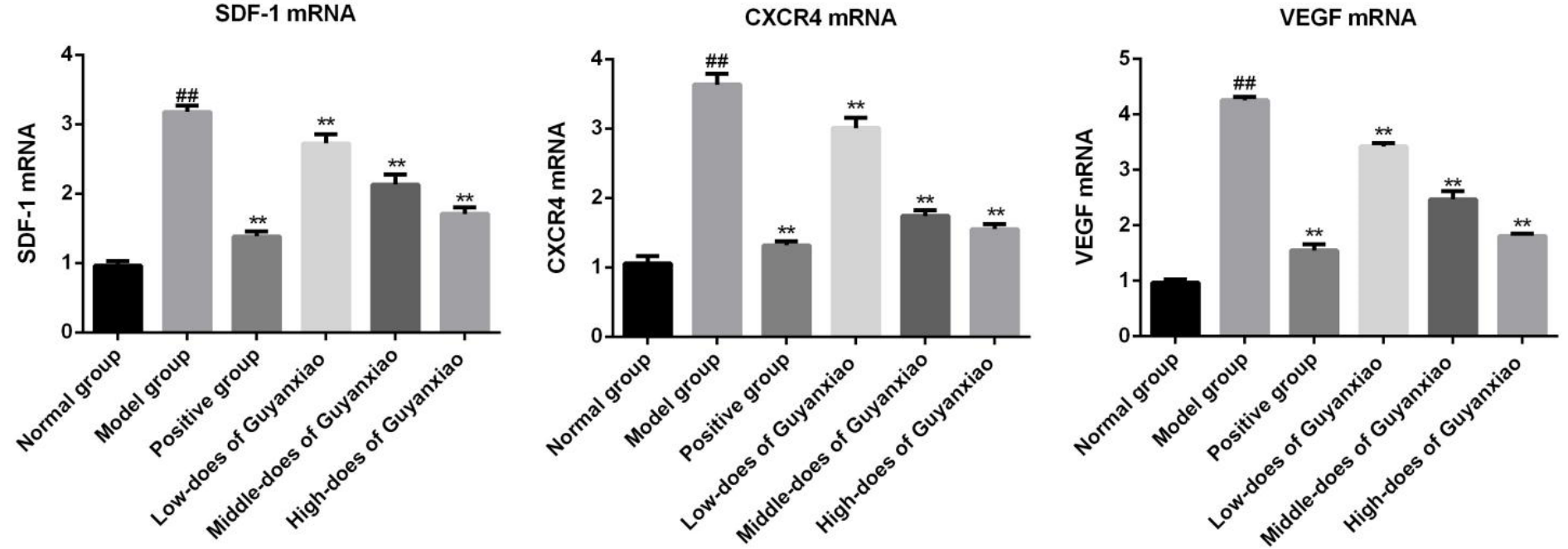

Figure 3

SDF-1, CXCR4, VEGF, IL-1 $\beta$ mRNA expression of chondrocytes in each group Note: Compared with the positive group, $\triangle \triangle P>0.05$

Figure 4

SDF-1, CXCR4 and VEGF protein bands in chondrocytes of each group following Western Blot analysis Note: A Normal group; B Model group; C Positive group; D Low-dose of Guyanxiao formula; E Middle-dose of Guyanxiao formula; F High-dose of Guyanxiao formula 